\title{
BMJ Open Medication reconciliation as a medication safety initiative in Ethiopia: a study protocol
}

\author{
Alemayehu B Mekonnen, ${ }^{1,2}$ Andrew J McLachlan, ${ }^{1}$ Jo-anne E Brien, ${ }^{1}$ \\ Desalew Mekonnen, ${ }^{3}$ Zenahebezu Abay ${ }^{4}$
}

To cite: Mekonnen AB, McLachlan AJ, Brien JE, et al. Medication reconciliation as a medication safety initiative in Ethiopia: a study protocol. BMJ Open 2016;6: 012322. doi:10.1136/bmjopen-2016012322

- Prepublication history and additional material is available. To view please visit the journal (http://dx.doi.org/ 10.1136/bmjopen-2016012322).

Received 17 April 2016 Revised 16 August 2016 Accepted 25 October 2016

CrossMark

\begin{abstract}
${ }^{1}$ Faculty of Pharmacy, University of Sydney, Sydney, New South Wales, Australia ${ }^{2}$ School of Pharmacy, University of Gondar, Gondar, Ethiopia

${ }^{3}$ Department of Internal Medicine, Addis Ababa University, Addis Ababa, Ethiopia

${ }^{4}$ Department of Internal Medicine, University of Gondar, Gondar, Ethiopia
\end{abstract}

Correspondence to A B Mekonnen; aber5592@uni.sydney.edu.au

\section{ABSTRACT}

Introduction: Medication related adverse events are common, particularly during transitions of care, and have a significant impact on patient outcomes and healthcare costs. Medication reconciliation (MedRec) is an important initiative to achieve the Quality Use of Medicines, and has been adopted as a standard practice in many developed countries. However, the impact of this strategy is rarely described in Ethiopia. The aims of this study are to explore patient safety culture, and to develop, implement and evaluate a theory informed MedRec intervention, with the aim of minimising the incidence of medication errors during hospital admission.

Methods and analyses: The study will be conducted in a resource limited setting. There are three phases to this project. The first phase is a mixed methods study of healthcare professionals' perspectives of patient safety culture and patients' experiences of medication related adverse events. In this phase, the Hospital Survey on Patient Safety Culture will be used along with semi-structured indepth interviews to investigate patient safety culture and experiences of medication related adverse events. The second phase will use a semi-structured interview guide, designed according to the 12 domains of the Theoretical Domains Framework, to explore the barriers and facilitators to medication safety activities delivered by hospital pharmacists. The third phase will be a single centre, before and after study, that will evaluate the impact of pharmacist conducted admission MedRec in an emergency department (ED). The main outcome measure is the incidence and potential clinical severity of medication errors. We will then analyse the differences in the incidence and severity of medication errors before and after initiation of an ED pharmacy service.

\section{INTRODUCTION}

\section{Patient safety initiatives}

Quality patient care is a priority issue in all healthcare sectors; however, clinical errors are known to compromise patient safety. ${ }^{1}$ Patient safety incidents gained attention after the works of pioneer US studies: the Harvard Medical Practice Study ${ }^{23}$ and the Institute of Medicine Report. ${ }^{4}$ In the USA, it has been

\section{Strengths and limitations of this study}

- This is the first study in Ethiopia that will assess the impact of a pharmacist led medication reconciliation service.

- This study is novel in that it uses a behavioural change theory for implementation of medication safety programmes.

- Multi-method exploration of patient safety issues will add substantial strength to our study.

- The sampling technique in both the interviews and survey may carry a risk of bias.

reported that $3.7 \%$ of all hospitalised patients experienced an adverse event, ${ }^{2}$ and medication errors alone resulted in 7000 deaths annually. ${ }^{4}$ Medication errors constitute the most common preventable cause of patient safety issues, and has been studied extensively in developed countries. ${ }^{2-6}$ Despite current advancements in healthcare, these incidents continue to pose a significant problem globally, ${ }^{7}$ and are the concern of many hospitalists and patient safety activists.

\section{Medication safety in African hospitals}

Patient injuries attributed to medication related adverse events are among the most common incidents in hospitals, ${ }^{2}$ and have important economic and humanistic consequences. Furthermore, given the morbidity profile and the high burden of malaria, HIV/AIDS and tuberculosis in Africa, along with the level of awareness and patient safety culture, the extent of medication related adverse events in African hospitals is thought to be higher than elsewhere in the world. ${ }^{8}$ For example, studies have shown that $1.5-6.5 \%$ of hospital admissions are attributed to adverse drug events (ADEs) ${ }^{9} \quad 10$ and $2.5-47 \%$ of inpatients encountered an ADE during their hospital stay. ${ }^{911}$ One-fifth to more than a half of the reported ADEs were severe events, ${ }^{10}{ }^{12-14}$ of 
which ADE related fatalities were reported in 0.07$2.9 \%$ of patient admissions to hospital. ${ }^{12} 1516$ However, up to half of the ADEs were due to medication errors and were preventable. ${ }^{10}$ The most reported types of medication errors in African healthcare settings were prescribing errors, occurring in 13-76\% of all prescriptions. ${ }^{17-20}$ Yet the extent of medication errors and ADEs have not been fully evaluated in African settings, ${ }^{8}$ and medication safety programmes designed to prevent them could represent the first step in improving patient safety.

\section{Medication reconciliation as a medication safety strategy}

More than half of the medication errors occurred at care transitions, when patients were admitted to, or discharged from, a hospital or transferred to the care of other healthcare professionals. ${ }^{21}$ Medication reconciliation (MedRec) as a tool for the prevention of these errors and consequent patient harm has been advocated internationally. ${ }^{22}{ }^{23}$ MedRec has been defined by the Institute for Healthcare Improvement as "the process of identifying the most accurate list of a patient's current medicines, including the name, dosage, frequency and route-and comparing them to the current list in use, recognising and documenting any discrepancies, thus resulting in a complete list of medications". ${ }^{22}$

Under the leadership of the WHO, patient safety programmes, including MedRec, have been implemented across a range of countries ${ }^{23-26}$ and taken up as healthcare policy. For example, MedRec has been recognised as a priority patient safety solution by the Australian Commission on Safety and Quality in Healthcare. ${ }^{26}$ Prior to MedRec being routinely practised in Australia, there was 1 omitted medicine from the medication chart for every 2 people at hospital admission. ${ }^{27}$ Also, previous studies showed that $60-80 \%$ of patients had a discrepancy in their medication history. ${ }^{28} 29$

Studies examining medication errors have been undertaken in many countries, including developing nations, ${ }^{30} 31$ in a range of settings, such as emergency units, ${ }^{32-38} \mathrm{critical} /$ intensive care units, ${ }^{39}$ and paediatric $^{40-42}$ and geriatric units. ${ }^{43-48}$ There is evidence that MedRec decreases the frequency of medication errors $^{49} 50$ and drug related readmissions. ${ }^{51} 52$

MedRec with various approaches has been employed to improve medication safety, including, but not limited to, technology assisted tools, ${ }^{53-55}$ use of standardised forms, ${ }^{34}$ collaborative models, ${ }^{33}{ }^{57}$ as well as patient engagement $^{58}$ and pharmacist led approaches. ${ }^{59} 60$ Previous studies have shown benefits from involving pharmacists in MedRec. ${ }^{59}{ }^{60}$ However, the impact of MedRec overall, as well as pharmacist led MedRec practice, has not yet been described in sub-Saharan Africa.

\section{Patient safety culture in the Ethiopian context}

Despite a lack of research, patient safety in Ethiopia is believed to be a serious concern. A previous local study $^{61}$ in paediatrics ward showed an incidence of 9.2 ADEs per 100 admissions, of which one-third were deemed preventable. As healthcare managers strive to improve the quality of patient care, there is a growing recognition of the importance of establishing a culture of patient safety. Developing a patient safety culture was one of the recommendations made by the Institute of Medicine $^{4}$ to assist hospitals in improving patient safety. According to the Agency of Healthcare Research and Quality (AHRQ) ${ }^{62}$ patient safety culture is described as an understanding of the values, beliefs and norms about what is important in an organisation and what attitudes and behaviours related to patient safety are supported, rewarded and expected. Thus it is important for healthcare organisations to assess their patient safety culture to gain a clear understanding of the patient safety aspects requiring urgent attention, identify the strengths and weaknesses of their safety culture ${ }^{63}$ and assist hospitals in identifying their existing patient safety problems. ${ }^{64}$ Studies on patient safety culture, mostly set in developed countries, ${ }^{63-66}$ have been published. However, there are no data about the current state of the patient safety culture in Ethiopian hospitals. Furthermore, no studies have specifically investigated implementation of MedRec services from a behavioural theory perspective, involving both barriers and facilitators of a wide range of behavioural determinants in the implementation of evidence based practice.

This project is a medication safety initiative focusing on MedRec at care transitions in Ethiopian public hospitals, and implementation of this service is guided by a multi-method approach consisting of three different but inter-related studies to inform our study objectives. Specifically, the aims of this study are: to explore healthcare professionals' views of patient safety issues, medical error, and event reporting and patients' experiences of medication related adverse events; to use a theoretical framework to help identify the barriers and facilitators to medication safety activities delivered by hospital pharmacists; and to evaluate a pharmacist led MedRec practice in one of the teaching hospitals in Ethiopia.

\section{METHODS AND ANALYSES}

\section{Study setting and period}

This is a multi-phased study that is being conducted in public hospitals in the Amhara region of Ethiopia. The Amhara region is one of nine regions of Ethiopia located in the northern part of the country. This region has an estimated total population of approximately 18 million, with the majority $(87.4 \%)$ being rural inhabitants. This region has 17 public hospitals, 520 health centres and 2941 health posts. ${ }^{67}$

There are three phases to this research project. Phases 1 and 2 are being conducted in 10 selected public hospitals in the Amhara region, including 4 teaching or referral hospitals (Gondar University, Felege Hiwot, Debre Markos and Debre Tabor) and 6 district hospitals 
(Metema, Debark, Chagni, Finoteselam, Woldiya and Enat). Phase 3 will be carried out in one teaching hospital (Gondar University Hospital (GUH)). The study started in February 2016 and will end in July 2017.

\section{Phase 1: a study of healthcare professionals' perspectives of patient safety culture and patients' experiences of medication related adverse events}

This is a mixed methods study consisting of a survey and qualitative research. The survey measures dimensional scores of the patient safety culture. Using a scale to quantify the scores for patient safety is, however, not explanatory. ${ }^{68}$ In addition, a shared decision between the patient and healthcare professional is central for a sustainable patient safety culture. Therefore, a survey supported by an indepth interview is well acknowledged as a meaningful assessment of patient safety culture through the eyes of healthcare professionals and patients. ${ }^{68}$

\section{Questionnaire study}

The survey aims to evaluate the patient safety culture of public hospitals in the Amhara region. The study focus is on public hospitals only as most of the population in the region use public hospitals. The study adopted the Hospital Survey on Patient Safety Culture (HSOPSC) developed by AHRQ ${ }^{69}$ HSOPSC has been widely used in assessing patient safety culture and has also been validated in non-US countries. ${ }^{64}{ }^{65}$ The survey consists of 42 items that measure 12 patient safety culture composites: communication openness, feedback and communication about errors, frequency of events reported, handovers and transitions, management support for patient safety, non-punitive response to error, organisational learning and continuous improvement, overall perceptions of patient safety, staffing, supervisor/manager expectations and actions promoting safety, and teamwork across and within units. Background characteristics of participants include questions related to job category, type of hospital (teaching/referral, district), years of working experience overall and in the current working area, work setting and working hours per week. The questionnaire is in English, as English is the main language of communication in Ethiopian hospitals. This questionnaire, together with the participant information statement, was distributed to conveniently selected healthcare professionals by the research team and required about $10-15 \mathrm{~min}$ to complete. These participants were recruited from the 10 hospitals in the Amhara region, and included physicians, nurses, pharmacists and paramedics (eg, technicians). The sample size was estimated to be 480 , by considering $95 \%$ CI, a $5 \%$ margin of error and $25 \%$ contingency for a nonresponse rate, and assuming that patient safety culture score was rated as excellent in $50 \%$ of respondents.

The response to each item in the questionnaire was assessed using a 5 point Likert scale, where 1 is 'strongly disagree' and 5 is 'strongly agree'. The patient safety grade (measured on a scale of excellent, very good, acceptable, poor and failing) and number of events reported were the other two outcome variables of interest. Currently, we are entering the collected data into SPSS v21, and data will be analysed when data entry is accomplished. The HSOPSC included both positively and negatively worded items. For easier interpretation of the results, $\mathrm{AHRQ}^{69}$ and other studies ${ }^{63-66}$ recommend the use of 'average positive' for calculating each item scores-that is, the percentage of positive responses for each item will be calculated, and negatively worded items will be reversed when computing per cent positive response. We will define areas of strength as items for which $75 \%$ of respondents answer positively and areas requiring improvement as those scoring $<50 \%$. ${ }^{62}$ Additionally, univariate and multivariate analyses will be conducted to examine statistical associations between independent characteristics and patient safety grade and number of events reported. The mean scores for each of the HSOPSC subscales are taken as dependent variables, and these will be tested against the independent variables, such as job characteristics (profession and qualification), department and type of hospital (teaching/ referral, district), work experience (career length, experience in the current unit/hospital) and workload (working hours).

\section{Indepth interview}

The qualitative part of the phase 1 investigation aims to assess patient safety strategies employed by those hospitals through indepth interviews with different stakeholders (healthcare professionals and patients) working in 10 hospitals in the Amhara region. The contact details of participants (healthcare professionals) were retrieved from the human resource office or related office of the respective hospitals. We are using purposeful sampling to identify the initial sample and then the remaining data collection is being aided by snowball sampling. We will invite healthcare professionals who are involved in the care of patients by letter or email, as appropriate. Patients who are in hospital at the time of data collection and were taking regular medications before admission will be invited for interview by a healthcare professional who is already a participant in this study. Next, we will contact patients for further invitation into the study. We will employ semi-structured interviews informed by the interview guide (see online supplementary additional file 1) for the collection of data. All interview guides have been translated from the English versions to the local language (Amharic) by two non-official translators who are native speakers and working in the healthcare industry, and validated by two of the research group (ABM, DM). Interview tools have been translated to foster faster communication and expression of ideas.

Before the interview, we will inform respondents about the aim of the interview, and those who consent will be given further details on the nature of the study to 
ensure that interviewees understand what is required of them. We will conduct face to face interviews at a time and place to suit the participants, and the interviews are expected to last approximately $30-60 \mathrm{~min}$. We will forward both open and close ended questions to interviewees to describe their experiences of medication safety issues and strategies employed to prevent medication related adverse events. We will encourage participants to reflect on their own experiences of medication related adverse events, and we will ask them to think about an example of a known medication related adverse event when answering questions.

The interviewer will use prompts when necessary to encourage further elaboration. We will give 50 Ethiopian birr to participants in appreciation of their time. All interviews will be conducted by an English/Amharic speaking investigator $(\mathrm{ABM})$. We will collect data from each of the two participant groups until a point of saturation is reached. We will record all interviews using audiotape with informed consent of participants. After data collection is completed, the principal investigator will carry out verbatim Amharic transcriptions of all interviews, which will then be translated into English, assigned a unique identifier and imported into a computer programme (Nvivo V10) for qualitative data analysis. Thematic analysis will then be carried out, and emerging topics will be identified as themes and sub-themes.

\section{Phase 2: barriers and facilitators to medication safety} activities delivered by hospital pharmacists

This is a qualitative study using focus group discussions (FGD) with hospital pharmacists working in selected public hospitals in the region to gather data on the barriers and facilitators to medication safety activities. We will employ FGDs in this phase because the interactive nature of focus groups is specifically important when group norms and cultural values of particular groups are of interest, and to explore the degree of consensus on a given topic, ${ }^{70}$ including implementation of an intervention to promote medication safety. Many factors can affect the adaptability of an evidence based intervention, and the success of implementation efforts depends on a careful assessment of barriers to, and facilitators of, the behaviour to be changed. ${ }^{71}$ A theory based identification of such factors provides a theoretically robust evidence base to inform implementation of an intervention. ${ }^{71}$ The underpinning theoretical model used in this study is the Theoretical Domains Framework (TDF).

\section{Theoretical Domains Framework}

Increasing the uptake of evidence into clinical practice and improving patient outcomes needs behaviour change. The TDF from health psychology provides the basis for such an approach, ensuring that a wide range of possible theoretical explanations for the behaviours can be considered. Built from 33 behavioural theories, the TDF was developed to make theories more accessible for implementation researchers. ${ }^{72}$ According to Michie $e t a l^{72}$ the TDF has 12 domains to explain behaviour change: (1) knowledge, (2) skills, (3) social/ professional role and identity, (4) beliefs about capabilities, (5) beliefs about consequences, (6) motivation and goals, (7) memory, attention and decision processes, (8) environmental context and resources, (9) social influences, (10) emotion regulation, (11) behavioural regulation and (12) nature of the behaviour. The TDF has been extensively used to identify barriers to change in clinical practice to develop interventions. ${ }^{73}{ }^{74}$ To justify implementation of pharmacist led MedRec, it will be critical to understand the perceived barriers and facilitators underlying the individual pharmacist's role in medication safety. Thus this study uses the TDF to develop a theory informed intervention aimed at improving medication safety of patients at hospital transitions.

\section{Focus group discussions}

In this study, FGDs will be guided by questions designed based on the TDF (table 1). For each of the 12 domains that could act as facilitators or barriers to current medication safety practices and a successful MedRec implementation, the authors developed several interview questions. The number of interview questions ranged between 2 and 5 for each of the 12 domains, for a total of 43 questions to cover a wide range of constructs assigned to each domain. The questions were initially drafted by one researcher (ABM) and then refined by health service researchers (AJM, JEB) and discussed by the research team to check for clinical relevance. The discussion questions will be pilot tested with at least two hospital pharmacists to assess clarity and focus, and revised accordingly.

The sample population will be all hospital pharmacists in the 10 public hospitals across the region. Pharmacists will be selected using a purposive sampling strategy augmented with snowball sampling. Participants will be recruited either by letter or email invitation. Participants willing to be interviewed by sending an email or by returning a signed consent form will be contacted. The principal investigator (ABM), who is experienced in qualitative study, will conduct and facilitate the FGD using the translated version (Amharic) of the topic guide. Pharmacists will be encouraged to talk about internal beliefs and attitudes that may hinder them from providing clinical pharmacy services, including medication safety roles. All discussion sessions will be audiotaped and recorded. Two of the researchers (ABM, ZA) will read all of the FGD Amharic transcripts, and will be translated into English. Transcripts will then be coded based on the 12 domains of the TDF, and thematic analysis of pharmacists' statements into the relevant theoretical domains will be performed. ${ }^{75}$ Briefly, the analysis will involve identifying contextualised brief statements related to the barriers and facilitators to medication safety activities, categorising statements 
Table 1 Interview guide questions for focus groups according to Michie's theoretical domains ${ }^{72}$

\begin{tabular}{|c|c|}
\hline Domains & Interview questions \\
\hline Knowledge & $\begin{array}{l}\text { Are there any hospital guidelines for pharmacists to deliver clinical pharmacy services? } \\
\text { What do you think the level of evidence is for these guidelines? } \\
\text { What do you know about medication reconciliation and review? } \\
\text { What do you think is the level of evidence for medication reconciliation and review? } \\
\text { Can you describe pharmacists' roles in medication safety activities? }\end{array}$ \\
\hline Skills & $\begin{array}{l}\text { Do you know how to deliver clinical pharmacy services? } \\
\text { Do you know how to deliver medication reconciliation and review services? } \\
\text { Is identification of medication related problems difficult for you? } \\
\text { Have you attended in-service training to deliver clinical pharmacy services? }\end{array}$ \\
\hline Social/professional role & $\begin{array}{l}\text { Is doing medication reconciliation and review compatible with your professional role? } \\
\text { Who is responsible for these services at your hospital? } \\
\text { Do you think hospital guidelines supports your professional roles as a pharmaceutical care } \\
\text { practitioners? }\end{array}$ \\
\hline Beliefs about capabilities & $\begin{array}{l}\text { How easy or difficult do you find performing clinical pharmacy activities? } \\
\text { What problems have you encountered? } \\
\text { How capable are you in performing medication reconciliation and review? } \\
\text { How confident are you that you can do these services despite difficulties? } \\
\text { How comfortable do you feel to undertake these services? }\end{array}$ \\
\hline Beliefs about consequences & $\begin{array}{l}\text { What are the likely positive/negative outcomes of reporting/communicating medication } \\
\text { related problems? } \\
\text { What are the costs of delivering medication reconciliation and review, and what are the } \\
\text { costs of the consequences of these services? } \\
\text { Are you concerned if these services are not provided at your hospital? } \\
\text { Do the benefits of doing these services outweigh the costs? } \\
\text { Does the evidence suggests that doing these services are beneficial? }\end{array}$ \\
\hline Motivation and goals & $\begin{array}{l}\text { How motivated are you to deliver medication reconciliation and review? } \\
\text { Are there incentives to provide these services? } \\
\text { Do you have any other hospital activity that hinders these services? }\end{array}$ \\
\hline $\begin{array}{l}\text { Memory, attention and decision } \\
\text { processes }\end{array}$ & $\begin{array}{l}\text { Will you consider providing medication reconciliation and review services? If so, how } \\
\text { frequently would you undertake this activity? } \\
\text { How much priority have you given to these services? }\end{array}$ \\
\hline $\begin{array}{l}\text { Environmental context and } \\
\text { resources }\end{array}$ & $\begin{array}{l}\text { To what extent do physical factors or resources facilitate or hinder delivering medication } \\
\text { reconciliation/review? } \\
\text { Are there competing tasks and time constraints? } \\
\text { Are the necessary resources available to undertake these services? } \\
\text { Do these services have advantages compared with the standard care? } \\
\text { Do government and local authorities provide sufficient support for these services? }\end{array}$ \\
\hline Social influences & $\begin{array}{l}\text { Are clinical pharmacy services in the hospital well acknowledged by other healthcare } \\
\text { professionals? } \\
\text { Do hospital managers acknowledge your role? } \\
\text { Is there any obstruction to these activities in your hospital? } \\
\text { Have you observed others providing these clinical services? }\end{array}$ \\
\hline Emotion & $\begin{array}{l}\text { What things worry you the most in providing medication reconciliation/review services? } \\
\text { To what extent do emotional factors facilitate or hinder these services? }\end{array}$ \\
\hline Behavioural regulation & $\begin{array}{l}\text { Have you received feedback from other healthcare professionals regarding these } \\
\text { services? } \\
\text { What intimal steps are needed to deliver these services? }\end{array}$ \\
\hline Nature of the behaviours & $\begin{array}{l}\text { What do you currently do? } \\
\text { How long are the changes going to take? } \\
\text { Are there any systems in place for sustainable long term changes? }\end{array}$ \\
\hline
\end{tabular}

into TDF domains and mapping the underlying theoretical constructs within domains. Both inductive and deductive approaches will be used so as not to miss any themes. To assess agreement between two researchers, all extracted themes and sub-themes will be reviewed in a meeting, and disagreements will be solved through consensus.
Phase 3: evaluation of the impact of a pharmacist led MedRec service. Single site before and after study

This phase of the project is the main objective of this study, and the aim is to investigate the impact of a pharmacist led MedRec service on the rate and incidence of unintentional medication discrepancies in an emergency ward of the GUH, Ethiopia. GUH is located 
in Gondar town in the Amhara regional state. It is the primary hospital in the northwest region of Ethiopia. GUH provides specialised health services through its medical and other clinical and diagnostic departments for a catchment population of approximately 5 million people.

The sample size calculation is based on the prevalence of medication errors in previous local studies, which was identified as 52-58\% of all prescriptions. ${ }^{17}{ }^{76}$ Assuming a reduction in medication errors from $55 \%$ to $45 \%, 80 \%$ power, 5\% significance level (two sided), we required 127 patients, 51 for the baseline and 76 for the intervention. Hospital discharge statistics showed that this sample size would be achievable in 3 months. A baseline assessment of medication discrepancies will thus be conducted for 1 month during hospital admission. Medication discrepancies are defined as one or more differences in dosage, frequency, drug or route of administration, as described by the Institute for Healthcare Improvement, ${ }^{22}$ between the current and previous medication (s) a patient was taking. A pharmacist led MedRec intervention will then be carried out prospectively for 2 months. Inclusion criteria will be patients >18 years of age, hospitalised for at least 24 hours and taking at least two home/regular medications on admission.

The standard practice in the current department involves physicians taking the patient's medication history using patient provided information; however, hospital pharmacists do not participate in medication history taking and prescription review in the emergency department. The intervention will involve use of the best possible medication history (BPMH), ${ }^{77}$ which is based on a structured interview with the patient about medication use and retrieving other sources of medication history, including discharge and referral letters, the patient's own medicines and carrier interview. One pharmacy staff member will be trained in the techinques of how to get the BPMH by a research pharmacist (ABM). MedRec will be conducted after patients are informed of the study and give written consent. Medication use will be documented within 24 hours of patient admission through a data collection tool prepared for the purpose of this study (see online supplementary additional file 2). The pharmacist will then compare the BPMH with the admission prescription order of the patient issued by the physician in charge.

All identified discrepancies will be brought to the attention of the physician at admission and verification of these discrepancies will be made-that is, intentional versus unintentional changes to medications. Intentional medication discrepancies are medication changes due to new clinical status of the patient, and are clinically justifiable but not documented in the patient's medical record. Thus only unintentional medication discrepancies (also called as medication errors) will be reported. The main outcome measure is the incidence of medication errors and the potential clinical severity of such errors. The potential clinical severity of medication errors will be judged by a consensus between a clinical pharmacist and a physician using a tool developed by Cornish et al. ${ }^{78}$ Descriptive statistics will be used to characterise the types of medication errors and the $\chi^{2}$ test will be used to analyse differences in the incidence and severity of medication errors between the baseline and intervention groups. Statistical significance is set at $\mathrm{p}<0.05$.

\section{Ethics and dissemination}

The study protocol was approved by the University of Sydney Human Research Ethics Committee (project No: $2015 / 818$ ) and the institutional review board of the University of Gondar, Ethiopia (O/V/P/RCS/05/624/ 2016). The data from this study will be disseminated to researchers, clinicians and health planners in peer reviewed health journals and conference publications. One or more meetings will be held locally to give feedback to participants and contributors to the study.

\section{DISCUSSION}

Patient safety in general, and medication safety in particular, has become a matter of growing interest and increasing priority for hospital managers. A safety culture is a necessary prerequisite for the improvement of patient safety. However, it is unclear how healthcare professionals and patients in Ethiopia perceive patient safety. This study will describe the views of healthcare professionals in hospitals about patient safety culture and patients' experiences of medication related adverse events, and use a behavioural change theory to implement a MedRec service. MedRec is a complex intervention conducted across a range of hospital transitions, and will therefore apply the TDF to a behaviour that is complex-for example, involving multiple procedures and conducted by various healthcare professionals.

This study has several strengths. This is the first study in Ethiopia that will assess the impact of a pharmacist led MedRec service, and is also novel in that it uses a theory informed implementation of this new practice as a medication safety strategy. The use of multi-method for the exploration of patient safety culture and practice will add substantial strength to our study. Use of behavioural theory that is commonly used in implementation studies will allow us to identify and select potentially relevant domains to target behaviour in detail. This study will contribute to the knowledge base by providing more evidence to confirm the importance of MedRec in improving the Quality Use of Medicines when patients are admitted to hospital. The challenge of designing quality improvement projects in resource limited settings is workload among the staff. We hope the data from this study will help develop evidence based medication safety interventions to strengthen the capacity and performance of hospital pharmacists in settings where resources are scarce. This study is not without limitations. The 
sampling technique in the qualitative study may carry a risk of bias by recruiting participants who may have similar opinions and experiences. To minimise this, participants will be requested to nominate other participants who have different experiences and practice in medication safety. Moreover, we will use an iterative process for data collection and analysis for the qualitative studies in phases 1 and 2 until we are sure that there are no new ideas emerging.

Acknowledgements This project was conducted as a partial fulfilment for a $\mathrm{PhD}$ in pharmacy (health services and patient safety theme) for ABM. He is supported by the University of Sydney International Students' Scholarship.

Contributors ABM, AJM and JEB contributed to the conception and design of the study. ABM drafted the first protocol. JEB and $A B M$ refined the study protocol with contributions from all co-authors (AJM, DM and ZA). All authors read and approved the final manuscript.

\section{Competing interests None declared.}

Ethics approval The study protocol was approved by the University of Sydney Human Research Ethics Committee (project No: 2015/818) and the institutional review board of the University of Gondar, Ethiopia (O/N/P/RCS/ 05/624/2016).

Provenance and peer review Not commissioned; externally peer reviewed.

Open Access This is an Open Access article distributed in accordance with the Creative Commons Attribution Non Commercial (CC BY-NC 4.0) license, which permits others to distribute, remix, adapt, build upon this work noncommercially, and license their derivative works on different terms, provided the original work is properly cited and the use is non-commercial. See: http:// creativecommons.org/licenses/by-nc/4.0/

\section{REFERENCES}

1. Benjamin DM. Reducing medication errors and increasing patient safety: case studies in clinical pharmacology. $J$ Clin Pharmacol 2003;43:768-83.

2. Brennan TA, Leape LL, Laird NM, et al. Incidence of adverse events and negligence in hospitalized patients. Results of the Harvard Medical Practice Study I. N Engl J Med 1991;324:370-6.

3. Leape LL, Brennan TA, Laird N, et al. The nature of adverse events in hospitalized patients: Results of the Harvard Medical Practice Study II. N Engl J Med 1991;324:377-84.

4. Institute of Medicine (US) Committee on Quality of Health Care in America; Kohn LT, Corrigan JM, Donaldson MS, eds. To Err is Human: Building a Safer Health System. Washington (DC): National Academies Press (US); 2000. 2, Errors in health care: A leading cause of death and injury. https://www.ncbi.nlm.nih.gov/books/ NBK225187/ (accessed 10 Nov 2016).

5. Roughead EE, Semple SJ. Medication safety in acute care in Australia: where are we now? Part 1: a review of the extent and causes of medication problems 2002-2008. Aust NZ Health Policy 2009;6:18.

6. Bouvy JC, De Bruin ML, Koopmanschap MA. Epidemiology of adverse drug reactions in Europe: a review of recent observational studies. Drug Saf 2015;38:437-53.

7. Clancy CM. Ten years after to err is human. Am J Med Qual 2009;24:525-8.

8. Isah AO, Pal SN, Olsson S, et al. Specific features of medicines safety and pharmacovigilance in Africa. Ther Adv Drug Saf 2012;3:25-34.

9. Tumwikirize WA, Ogwal-Okeng JW, Vernby A, et al. Adverse drug reactions in patients admitted on internal medicine wards in district and regional hospitals in Uganda. Afr Health Sci. 2011;11:72-8.

10. Mehta U, Durrheim DN, Blockman M, et al. Adverse drug reactions in adult medical inpatients in a South African hospital serving a community with a high HIV/AIDS prevalence: prospective observational study. Br J Clin Pharmacol 2008;65:396-406.

11. Letaief M, El Mhamdi S, El-Asady R, et al. Adverse events in a Tunisian hospital: results of a retrospective cohort study. Int J Qual Health Care 2010;22:380-5.
12. Benkirane RR, Abouqal R, Haimeur CC, et al. Incidence of adverse drug events and medication errors in intensive care units: a prospective multicenter study. J Patient Saf 2009;5:16-22.

13. Jennane N, Madani N, Oulderrkhis R, et al. Incidence of medication errors in a Moroccan medical intensive care unit. Int Arch Med 2011;4:32.

14. Oshikoya KA, Chukwura H, Njokanma OF, et al. Incidence and cost estimate of treating pediatric adverse drug reactions in Lagos, Nigeria. Sao Paulo Med J 2011;129:153-64.

15. Benkirane R, Pariente A, Achour S, et al. Prevalence and preventability of adverse drug events in a teaching hospital: a cross-sectional study. EMHJ 2009;15:1145-55.

16. Mouton J, Njuguna C, Stewart A, et al. Mortality from adverse drug reactions in adult medical inpatients at four hospitals in South Africa: a cross sectional survey. Br J Clin Pharmacol 2015;80:818-26.

17. Agalu A, Ayele $\mathrm{Y}$, Bedada W, et al. Medication prescribing errors in the intensive care unit of Jimma University Specialized Hospital, Southwest Ethiopia. J Multidiscip Healthc 2011;4:377-82.

18. Ajemigbitse AA, Omole MK, Erhun WO. An assessment of the rate, types and severity of prescribing errors in a tertiary hospital in southwestern Nigeria. Afr J Med Med Sci 2013;42:339-46.

19. Oshikoya KA, Ojo OI. Medication errors in paediatric outpatient prescriptions of a teaching hospital in Nigeria. Nig Q J Hosp Med 2007;17:74-8.

20. Yinusa W. Prescription error in an orthopaedic practice. Niger Postgrad Med J 2004;11:37-9.

21. Rozich JD, Howard RJ, Justeson JM, et al. Standardization as a mechanism to improve safety in health care. Jt Comm J Qual Saf 2004;30:5-14.

22. Institute for Healthcare Improvement. Medication reconciliation review. http://www.ihi.org/resources/Pages/Tools/Medication ReconciliationReview.aspx (accessed 16 Nov 2015).

23. Joint Commission on Accreditation for Healthcare Organizations, 2006. National patient safety goals. https://www.jointcommission.org/ improving_americas_hospitals_the_joint_commissions_annual_ report_on_quality_and_safety_-_2006/(accessed 16 Nov 2015).

24. National Instutiute of Clinical Excellence/ National Patient Safety Agency. NICE/NPSA Technical patient safety solutions for medicines reconciliation on admission of adults to hospital. London, 2007. http://www.nice.org.uk/PSG001 (accessed 16 Nov 2015).

25. Canadian Council on Health Services Accreditation. CCHSA Patient Safety Goals and Required Organizational Practices. Ottawa, 2004. http://www.accreditation.ca (accessed 16 Nov 2015).

26. Australian Commission on Safety and Quality in Healthcare. Medication reconciliation. http://www.safetyandquality.gov.au/ our-work/medication-safety/medication-reconciliation/ (accessed 16 Nov 2015).

27. Australian Council on Safety and Quality in Health Care. Second national report on improving patient safety: improving medication safety. Canberra: Australian Council on Safety and Quality in Health Care, 2002.

28. Tompson AJ, Peterson GM, Jackson SL, et al. Utilizing community pharmacy dispensing records to disclose errors in hospital admission drug charts. Int J Clin Pharmacol Ther 2012;50:639-46.

29. Vasileff HM, Whitten LE, Pink JA, et al. The effect on medication errors of pharmacists charting medication in an emergency department. Pharm World Sci 2009;31:373-9.

30. Abuyassin BH, Aljadhey $\mathrm{H}$, Al-Sultan $\mathrm{M}$, et al. Accuracy of the medication history at admission to hospital in Saudi Arabia. Saudi Pharm J 2011;19:263-7.

31. Hassali MA, Al-Haddad M, Shafie AA, et al. Perceptions among general medical practitioners toward implementation of medication reconciliation program for patients discharged from hospitals in Penang, Malaysia. J Patient Saf 2012;8:76-80.

32. Caglar S, Henneman PL, Blank FS, et al. Emergency department medication lists are not accurate. J Emerg Med 2011;40:613-16.

33. De Winter S, Spriet I, Indevuyst C, et al. Pharmacist-versus physician-acquired medication history: a prospective study at the emergency department. Qual Saf Health Care 2010;19:371-5.

34. De Winter S, Vanbrabant P, Spriet I, et al. A simple tool to improve medication reconciliation at the emergency department. Eur $J$ Intern Med 2011;22:382-5.

35. Hummel J, Evans PC, Lee H. Medication reconciliation in the emergency department: opportunities for workflow redesign. Qual Saf Health Care 2010;19:531-5.

36. Boyce LE, Knight AT. Audit of general practitioner medication lists for older patients at a regional hospital. J Pharm Pract and Res 2013;43:105-8.

37. Mazer M, Deroos F, Hollander JE, et al. Medication history taking in emergency department triage is inaccurate and incomplete. Acad Emerg Med 2011;18:102-4. 
38. Mills PR, McGuffie AC. Formal medicine reconciliation within the emergency department reduces the medication error rates for emergency admissions. Emerg Med J 2010;27:911-15.

39. Hatch JB, Schulz L, Fish JT. Stress ulcer prophylaxis: reducing non-indicated prescribing after hospital discharge. Ann Pharmacother 2010;44:1565-71.

40. Rappaport DI, Collins B, Koster A, et al. Implementing medication reconciliation in outpatient pediatrics. Pediatrics 2011;128:e1600-7.

41. Stone BL, Boehme S, Mundorff MB, et al. Hospital admission medication reconciliation in medically complex children: an observational study. Arch Dis Child 2010;95:250-5.

42. Terry DR, Solanki GA, Sinclair AG, et al. Clinical significance of medication reconciliation in children admitted to a UK pediatric hospital: observational study of neurosurgical patients. Paediatr Drugs 2010;12:331-7.

43. Gizzi L, Slain D, Hare J, et al. An assessment of a pharmacy-based enhancement to the hospital medication reconciliation process. ASHP Midyear Clinical Meeting. 2009.

44. Gizzi LA, Slain D, Hare JT, et al. Assessment of a safety enhancement to the hospital medication reconciliation process for elderly patients. Am J Geriatr Pharmacother 2010;8:127-35.

45. Perennes $M$, Carde $A$, Nicolas $X$, et al. [Medication reconciliation: an innovative experience in an internal medicine unit to decrease errors due to inacurrate medication histories]. Presse Med 2012;41: e77-86.

46. Steurbaut S, Leemans L, Leysen T, et al. Medication history reconciliation by clinical pharmacists in elderly inpatients admitted from home or a nursing home. Ann Pharmacother 2010;44: 1596-603.

47. Stitt DM, Elliott DP, Thompson SN. Medication discrepancies identified at time of hospital discharge in a geriatric population. Am J Geriatr Pharmacother 2011;9:234-40.

48. Villanyi D, Fok M, Wong RY. Medication reconciliation: identifying medication discrepancies in acutely ill hospitalized older adults. Am J Geriatr Pharmacother 2011;9:339-44.

49. Geurts MM, van der Flier M, de Vries-Bots AM, et al. Medication reconciliation to solve discrepancies in discharge documents after discharge from the hospital. Int J Clin Pharm 2013;35:600-7.

50. Karapinar-Carkit F, Borgsteede SD, Zoer J, et al. Effect of medication reconciliation with and without patient counseling on the number of pharmaceutical interventions among patients discharged from the hospital. Ann Pharmacother 2009;43:1001-10.

51. Hellstrom LM, Bondesson A, Hoglund P, et al. Impact of the Lund Integrated Medicines Management (LIMM) model on medication appropriateness and drug-related hospital revisits. Eur J Clin Pharmacol 2011;67:741-52.

52. Jack BW, Chetty VK, Anthony D, et al. A reengineered hospital discharge program to decrease rehospitalization: a randomized trial. Ann Intern Med 2009;150:178-87.

53. Gimenez Manzorro A, Zoni AC, Rodriguez Rieiro C, et al. Developing a programme for medication reconciliation at the time of admission into hospital. Int J Clin Pharm 2011;33:603-9.

54. Schnipper JL, Liang CL, Hamann C, et al. Development of a too within the electronic medical record to facilitate medication reconciliation after hospital discharge. J Am Med Inform Assoc 2011;18:309-13.

55. Moore $\mathrm{P}$, Armitage $\mathrm{G}$, Wright $\mathrm{J}$, et al. Medicines reconciliation using a shared electronic health care record. J Patient Saf 2011:7:148-54.

56. Bedard $P$, Tardif $L$, Ferland $A$, et al. A medication reconciliation form and its impact on the medical record in a paediatric hospital. J Eval Clin Pract 2011:17:222-7.

57. Tessier EG, Henneman EA, Heelon M, et al. An evaluation of collaborative nurse-pharmacist intervention for improving the medication reconciliation process: impact on patient safety. ASHP Midyear Clinical Meeting. 2009:220.

58. Greenwald JL, Halasyamani L, Greene J, et al. Making inpatient medication reconciliation patient centered, clinically relevant and implementable: a consensus statement on key principles and necessary first steps. J Hospital Med 2010;5:477-85.
59. Mekonnen AB, McLachlan AJ, Brien JE. Effectiveness of pharmacist-led medication reconciliation programmes on clinical outcomes at hospital transitions: a systematic review and meta-analysis. BMJ Open 2016;6:e010003.

60. Mekonnen AB, McLachlan AJ, Brien JE. Pharmacy-led medication reconciliation programmes at hospital transitions: a systematic review and meta-analysis. J Clin Pharm Ther 2016;41:128-44.

61. Eshetie TC, Hailemeskel B, Mekonnen N, et al. Adverse drug events in hospitalized children at Ethiopian University Hospital: a prospective observational study. BMC Pediatr 2015;15:83.

62. Sorra JS, Nieva VF. Hospital Survey on Patient Safety Culture, AHRQ Publication No. 04-0041. Rockville, MD: Agency for Healthcare Research and Quality, 2004.

63. Smits M, Wagner C, Spreeuwenberg $\mathrm{P}$, et al. Measuring patient safety culture: an assessment of the clustering of responses at unit level and hospital level. Qual Saf Health Care 2009;18:292-6.

64. Deilkas E, Hofoss D. Psychometric properties of the Norwegian version of the Safety Attitudes Questionnaire (SAQ), generic version (short form 2006). BMC Health Serv Res 2008;8:191.

65. Smits M, Christiaans-Dingelhoff I, Wagner C, et al. The psychometric properties of the "Hospital Survey of Patient Safety Culture" in Dutch hospitals. BMC Health Serv Res 2008;8:230.

66. Wagner C, Smits M, Sorra J, et al. Assessing patient safety culture in hospitals across countries. Int $J$ Qual Health Care 2013;25:213-21.

67. Amhara Health Bureau, Minsitry of Health, Federal Democratic Republic of Ethiopia. http://www.moh.gov.et/amharahb (accessed 16 Nov 2015)

68. Liu C, Liu W, Wang Y, et al. Patient safety culture in China: a case study in an outpatient setting in Beijing. BMJ Qual Saf 2014;23:556-64.

69. Hospital survey on patient safety culture. Agency for Healthcare Research and Quality. http://www.ahrq.gov/professionals/ quality-patientsafety/patientsafetyculture/hospital/index.htm (accessed 16 Nov 2015)

70. Bussières $A E$, Patey $A M$, Francis $\mathrm{JJ}$, et al. Identifying factors likely to influence compliance with diagnostic imaging guideline recommendations for spine disorders among chiropractors in North America: a focus group study using the Theoretical Domains Framework. Implement Sci 2012;7:82.

71. French SD, Green SE, O'Connor DA, et al. Developing theory-informed behaviour change interventions to implement evidence into practice: a systematic approach using the theoretical domains framework. Implement Sci 2012;7:38.

72. Michie S, Johnston M, Lawton R, et al. Making psychological theory useful for implementing evidence based practice: a consensus approach. Qual Saf Health Care 2005;14:26-33.

73. Duncan EM, Francis JJ, Johnston M, et al. Learning curves, taking instructions, and patient safety: using a theoretical domains framework in an interview study to investigate prescribing errors among trainee doctors. Implement Sci 2012;7:86.

74. Mirbaha F, Shalviri G, Yazdizadeh B, et al. Perceived barriers to reporting adverse drug events in hospitals: a qualitative study using theoretical domains framework approach. Implementation Sci 2015;10:110.

75. Islam R, Tinmouth AT, Francis JJ, et al. A cross-country comparison of intensive care physicians' beliefs about their transfusion behaviour: a qualitative study using the theoretical domains framework. Implementation Sci 2012;7:93.

76. Zeleke A, Chanie T, Woldie M. Medication prescribing errors and associated factors at the pediatric wards of Dessie Referral Hospital, Northeast Ethiopia. Int Arch Med 2014;7:18.

77. Australian Commission on Safety and Quality in Healthcare. Taking a Best Possible Medication History. https://www.safetyandquality. gov.au/our-work/medication-safety/medication-reconciliation/ taking-a-best-possible-medication-history/ (accessed 23 Nov 2016).

78. Cornish PL, Knowles SR, Marchesano R, et al. Unintended medication discrepancies at the time of hospital admission. Arch Intern Med 2005;165:424-9. 